\title{
Effect of PTEN and $K A I 1$ gene overexpression on the proliferation, metastasis and radiosensitivity of ASPC-1 pancreatic cancer cells under hypoxic conditions
}

\author{
HONGYU LI ${ }^{1 *}$, JIANJUN LI ${ }^{2 *}$, XU LIU ${ }^{1}$, JIANG CHEN ${ }^{1}$, CHUNYAN WU $^{1}$ and XIAOZHONG GUO ${ }^{1}$ \\ ${ }^{1}$ Department of Gastroenterology, General Hospital of Shenyang Military Area, Shenyang, Liaoning 110840; \\ ${ }^{2}$ Department of Radiotherapy, The First Affiliated Hospital, China Medical University, Shenyang, Liaoning 110001, P.R. China
}

Received October 9, 2013; Accepted May 9, 2014

DOI: $10.3892 / \mathrm{mmr} .2014 .2404$

\begin{abstract}
The aim of the present study was to investigate the effects of PTEN and KAII gene overexpression on the proliferation, metastasis and radiosensitivity of ASPC-1 pancreatic cancer cells under hypoxic conditions. Recombinant vectors that overexpress PTEN and KAII genes were transfected into hypoxic ASPC-1 cells. Protein expression levels were detected by western blot analysis. An MTT cell growth curve assay and a colony-forming assay were used to analyze cell proliferation, and a Transwell assay was performed to evaluate the metastatic ability of the cells. Annexin V flow cytometry was used to determinate the apoptotic rate of $\mathrm{X}$-ray-treated ASPC-1 cells. Western blot analysis revealed that PTEN- and KAII-transfected ASPC-1 cells significantly upregulated $P T E N$ and $K A I I$ expression. The proliferation of hypoxic ASPC-1 cells was significantly suppressed by PTEN and KAII. Furthermore, PTEN and KAII overexpression inhibited the metastatic ability of hypoxic ASPC-1 cells. Following X-ray treatment, the percentage of apoptotic cells increased significantly in the ASPC-1 cells transfected with PTEN and KAIl, which demonstrated that hypoxic ASPC-1 cells were more radiosensitive due to PTEN and KAII overexpression. In conclusion, double overexpression of PTEN and $K A I I$ inhibited the proliferation and metastatic activity, and enhanced apoptosis induced by X-ray in ASPC-1 cells under hypoxic conditions, which indicates that PTEN and KAII double-expression may have valuable application in pancreatic cancer gene therapy.
\end{abstract}

Correspondence to: Professor Xiaozhong Guo, Department of Gastroenterology, General Hospital of Shenyang Military Area, 83 Wenhua Road, Shenhe Street, Shenyang, Liaoning 110840, P.R. China

E-mail: guoxiaozhong1962@163.com

${ }^{*}$ Contributed equally

Key words: PTEN, KAIl, hypoxia, pancreatic cancer, radiosensitivity

\section{Introduction}

Pancreatic cancer is an aggressive gastrointestinal tumor with a poor prognosis. Tissue hypoxia, due to uneven distribution of blood vessels, is a potent micro-environmental stress during tumor evolution and is a common feature of the majority of solid tumors (1). Several studies have demonstrated that hypoxia is associated with prevention of apoptosis, epithelial mesenchymal transition and angiogenesis of tumor cells, which promotes tumor proliferation and metastasis (2). Hypoxia may also reduce the efficacy of radiotherapy, chemotherapy and other therapeutic approaches (3).

The $K A I 1$ gene was originally isolated in prostate cancer cells (4). Reduced KAII mRNA expression levels were reported to correlate with the formation of metastases in pancreatic and colorectal cancer $(5,6)$. Phosphatase and tensin homolog deleted on chromosome 10 (PTEN)/phosphatidylinositol 3-kinase/Akt constitutes an important signaling pathway regulating multiple biological processes, including cell proliferation, apoptosis, metabolism and cell growth. Abrogated PTEN activity, through mutations, deletions or promoter methylation silencing, occurs at high frequency in numerous primary and metastatic human cancer types $(7,8)$.

The aim of the present study was to imitate the hypoxic environment in the ASPC-1 pancreatic cancer cell line, and investigate the effects of tumor suppressor gene PTEN and tumor metastasis suppressor gene KAII double-transfection on the proliferation, metastasis and radiosensitivity of ASPC-1 cells under hypoxic conditions. This may provide a theoretical foundation for controlling pancreatic cancer cell proliferation and metastasis via combined gene therapy.

\section{Materials and methods}

Materials. The following materials were used in the present study: ASPC-1 cell line (Shanghai Institute of Cell Biology, Chinese Academy of Sciences, Shanghai, China), Dulbecco's modified Eagle's medium (Gibco-BRL, Carlsbad, CA, USA), fetal calf serum (Gibco-BRL), Lipofectamine ${ }^{\mathrm{TM}} 2000$ (Invitrogen Life Technologies, Carlsbad, CA, USA), plasmid extraction kit (Qiagen, Hilden, Germany), PTEN antibody (Abcam, Cambridge, MA, USA), KAI1 antibody (Santa Cruz 
Biotechnology, Inc., Santa Cruz, CA, USA), MTT (Biyuntian, Shanghai, China), Giemsa (Biyuntian), Transwell chamber system (Corning Inc, Acton, MA, USA), Annexin V Apoptosis Detection kit (Chemicon, Temecula, CA, USA). The pEAK8 plasmid carrying the PTEN gene and adenovirus carrying the $K A I 1$ gene were prepared according to procedures described previously $(9,10)$.

Western blot analysis of PTEN and KAII protein overexpression in ASPC-1 cells under hypoxic conditions. The hypoxic environment was imitated by continuous mechanical ventilation with $1 \% \mathrm{O}_{2}, 5 \% \mathrm{CO}_{2}$ and $94 \% \mathrm{~N}_{2}$ in a completely closed square box. ASPC-1 cells in logarithmic growth phase were cultured under hypoxic conditions for one week. Subsequent to cell proliferation and division, the cells were seeded into 6-well plates and transfected with pEAK8 plasmids carrying the PTEN gene and adenoviruses carrying the KAIl gene using Lipofectamine 2000. Following screening and further cultivation of the recombinant cells, $2 \times 10^{6}$ cells were collected by trypsin and rinsed with phosphate-buffered saline (PBS) twice. The cell total protein was denatured at $95^{\circ} \mathrm{C}$ and quantified using the Bradford assay. A total of $50 \mu \mathrm{g}$ denatured protein was separated on a $12 \%$ polyacrylamide gel by electrophoresis and transferred to nitrocellulose membranes. The primary antibodies (PTEN and KAII antibody) and secondary antibodies [goat anti-mouse IgG-horseradish peroxidase (HRP) and goat anti-rabbit IgG-HRP antibodies] were successively incubated. The blotted membranes were treated using the SuperSignal West Dura Extended Duration Substrate (Pierce Biotechnology Inc., Rockford, IL, USA) and signals were detected using a Las-4000 mini CCD camera (GE Healthcare, Buckinghamshire, UK). Enhanced chemiluminescence was used to develop the immunoblots. GAPDH served as an internal control to normalize PTEN and KAII expression levels.

MTT assay of ASPC-1 cell proliferation following double transfection with PTEN and KAII genes under hypoxic conditions. The ASPC-1 cells double-transfected by PTEN and KAII genes and the control cells were cultured under hypoxic conditions for 1 week and then cultured in 96-well plates $\left(2 \times 10^{3}\right.$ cell/well). MTT $(5 \mathrm{mg} / \mathrm{ml})$ was added to the wells (10 $\mu \mathrm{l} /$ well) on day two following transfection and the plate was incubated at $37^{\circ} \mathrm{C}$ for $4 \mathrm{~h}$. The supernatants in the wells were removed and replaced with dimethyl sulfoxide (100 $\mu \mathrm{l} /$ well) for $5 \mathrm{~min}$. The optical density (OD) value of each well was measured using a microculture plate reader (eLX-800; BioTek Instruments Inc., Winooski, VT, USA) at $490 \mathrm{~nm}$. MTT detection was performed on five consecutive days and five parallel wells were designed at each time point. The cell proliferation curve was drawn using time as the $\mathrm{X}$-axis and the $\mathrm{OD}$ value at A490 nm as the Y-axis.

Tumor colony-forming assay of ASPC-1 cells following double transfection with PTEN and KAII genes under hypoxic conditions. The ASPC-1 cells double-transfected by PTEN and $K A I 1$ genes and the control cells were cultured under hypoxic conditions for 1 week and then transferred to 6-well plates (200 cells/well) with three parallel wells in each group. The cells were further cultured for another 14 days and changed with fresh medium every 3-4 days. The cells were rinsed twice at the end of the experiment and fixed in paraformaldehyde, followed by Giemsa staining for $10 \mathrm{~min}$. Subsequent to washing with deionized water three times, the tumor colonies were counted in each well and images of the colonies were captured using a microscope (IX51; Olympus, Tokyo, Japan).

Transwell assay of ASPC-1 cells following double transfection with PTEN and KAIl genes under hypoxic conditions. A transwell assay was performed according to the manufacturer's instructions. Subsequently, 30\% fetal calf serum medium was added to the lower chamber. Serum-free suspensions of ASPC-1 double-transfected cells and control cells were prepared and $2 \times 10^{4}$ cells were seeded into the upper chamber and incubated for $8 \mathrm{~h}$ in the incubator. The small chamber was turned upside-down and placed on absorbent paper to air-dry the medium. Non-invasive cells in the upper chamber were gently removed with a cotton swab and stained with Giemsa for $30 \mathrm{~min}$, then, subsequent to rinsing several times, images of the cells were captured through microscopes (IX51; Olympus). The cells were then dissolved in $10 \%$ acetic acid to measure the OD value at $570 \mathrm{~nm}$ using a microplate reader (BioTek Instruments Inc.).

Annexin $V$ flow cytometric assay of the apoptotic rate of $X$-ray-treated ASPC-1 cells. The ASPC-1 double-transfected cells and the control cells were cultured under hypoxic conditions and seeded into 6-well plates with three parallel wells for each group. When the cells reached $90 \%$ confluency, they were irradiated once at 8 Gy radiation dose (dose rate $2 \mathrm{~Gy} / \mathrm{min}$ ) by using an X-ray irradiator (MBR-1520R; Hitachi, Tokyo, Japan). The cells were cultured for a further $24 \mathrm{~h}$ and trypsinized. Following centrifugation and washing the cell precipitation with $\mathrm{PBS}$, the cells were resuspended in $0.5 \mathrm{ml}$ $1 \mathrm{X}$ binding buffer, $5 \mathrm{ml}$ Annexin V-APC (BD Biosiences, Franklin Lakes, NJ, USA) at $1 \times 10^{6}$ cells $/ \mathrm{ml}$. The cells were incubated for $15 \mathrm{~min}$ at room temperature and then analyzed by flow cytometry $\left(\right.$ Cytomics $^{\mathrm{TM}}$ FC500; Beckman Coulter, Miami, FL, USA).

Statistical analysis. All data are expressed as the mean \pm standard deviation. Data were analyzed using SPSS 16.0 software (SPSS, Inc., Chicago, IL, USA). The differences between groups were assessed with Student's paired t-test and $\mathrm{P}<0.05$ was considered to indicate a statistically significant difference.

\section{Results}

PTEN and KAIl protein expression efficiency. Subsequent to transfection of the ASPC-1 cells with the PTEN and KAII genes, the expression levels of PTEN and $K A I 1$ protein were significantly increased (Fig. 1). However, no significant differences were detected in PTEN and KAII protein expression levels between the control cells transfected with empty vector and the ASPC-1 blank control cells.

ASPC-1 cell growth curve following double-transfection with PTEN and KAII under hypoxic conditions. The ASPC-1 cells were separately co-transfected with PTEN and KAII overexpression vectors or empty vectors, and an MTT assay was performed to detect the OD value in each group 1-5 days 
A

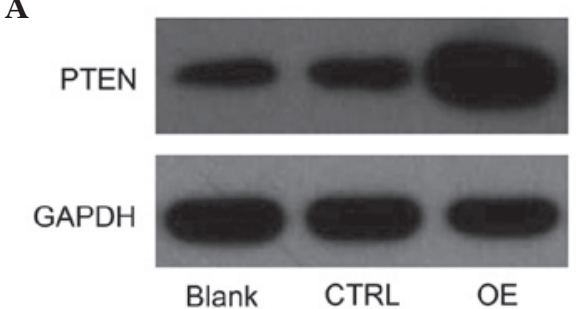

B

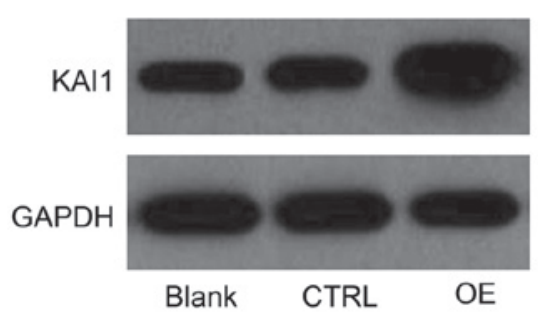

Figure 1. (A) PTEN and (B) KAI1 protein expression efficiency in ASPC-1 pancreatic cancer cells, analyzed by western blotting. Blank, blank control; CTRL, negative control transfected with empty vector; OE, cells double-transfected with PTEN and KAII genes.

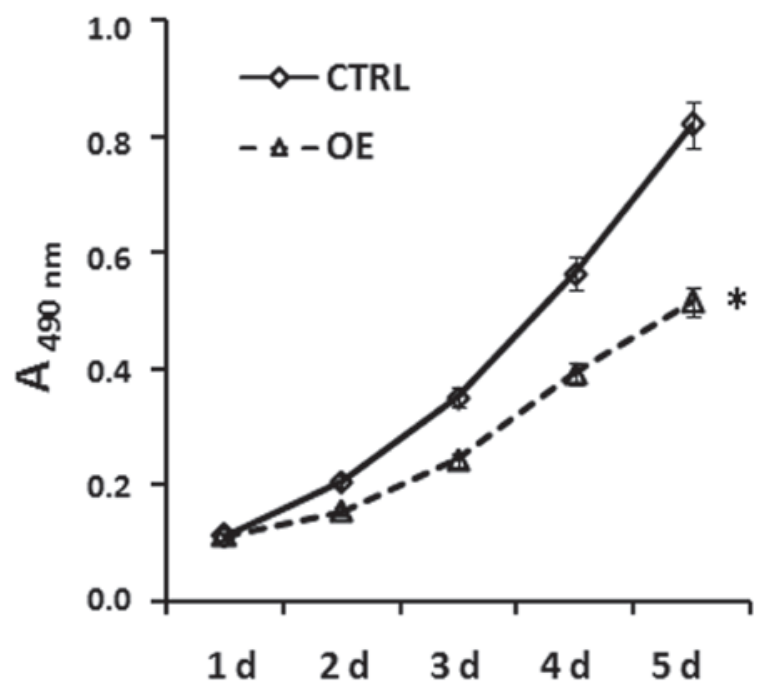

Figure 2. Proliferation of ASPC-1 pancreatic cancer cells under hypoxic conditions. CTRL, negative control cells transfected with empty vector; OE, cells double-transfected with PTEN and KAII genes; "P $<0.05$.

after transfection. Statistical analysis found that the proliferation rate of ASPC-1 cells co-transfected with PTEN and KAII over-expression vectors was significantly reduced, compared with that of the control group (Fig. 2). Notably, the OD values at 2-5 days after transfection were significantly lower than those in the control cells transfected with empty vectors $(\mathrm{P}<0.05)$.

Tumor colony-forming assay of ASPC-1 cells following double transfection with PTEN and KAII genes under hypoxic conditions. The results of the tumor colony-forming assay are shown in Fig. 3A. The tumor colony-forming ability was significantly inhibited in ASPC-1 cells transfected with PTEN and KAII genes compared with the ASPC-1 cells transfected with the empty vector. Giemsa staining and counting further confirmed that the number of tumor cells forming

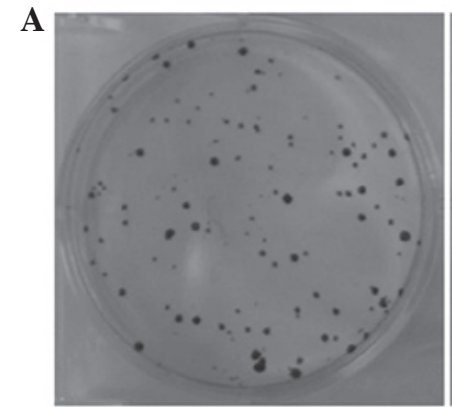

CTRL

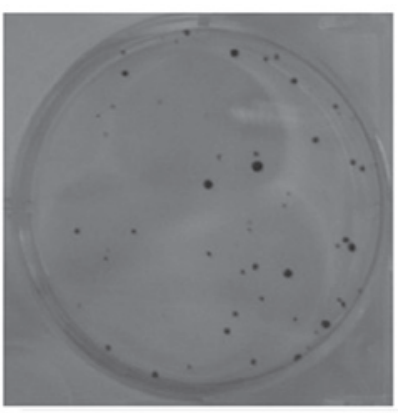

OE
B

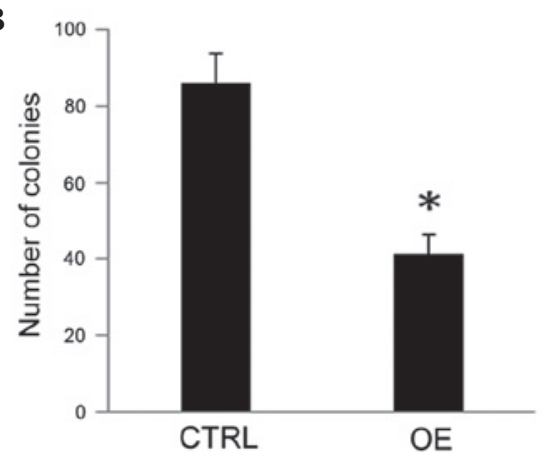

Figure 3. Colony-forming ability of ASPC-1 pancreatic cancer cells under hypoxic conditions. (A) Colony formation was observed under a microscope 2 weeks after incubation. (B) The number of colonies was calculated. Representative results from three independent experiments. CTRL, cells transfected with empty vector (negative control); OE, cells double-transfected with PTEN and KAII genes; ${ }^{*} \mathrm{P}<0.05$, compared with control.

tumor colonies with $>50$ cells per colony was significantly reduced $(\mathrm{P}<0.05$; Fig. 3B). The number of cells in single clones of ASPC-1 cells transfected with PTEN and KAII genes was significantly reduced compared with that of the control group $(\mathrm{P}<0.05)$.

Transwell assay of ASPC-1 cells following double transfection with PTEN and KAII genes under hypoxic conditions. In the present study, the tumor cell migration ability of the cells was evaluated using a Transwell assay. Giemsa staining of the migrated metastatic cells in the Transwell chamber revealed that the migratory ability of ASPC-1 cells transfected with PTEN and KAII genes was significantly reduced compared with that of the ASPC-1 cells transfected with empty vector (Fig. 4A). The cell migratory rate was calculated by the ratio between the OD value of migrated cells at the bottom of the chamber and the OD value of cells when seeded. This cell migratory rate was significantly reduced following double-transfection of ASPC-1 cells with the PTEN and KAII genes $(\mathrm{P}<0.05$; Fig. 4B).

Annexin $V$ flow cytometric assay of the apoptotic rate of $X$-ray-treated ASPC-1 cells. Using Annexin V staining of the cell membrane as an apoptotic index, the apoptotic rate of ASPC-1 cells double-transfected with PTEN and KAII genes or ASPC-1 cells transfected with empty vector was analyzed by flow cytometry (Fig. 5A). The results revealed that the apoptotic rate of ASPC-1 cells transfected with PTEN and $K A I 1$ genes was significantly increased, when compared with 
A

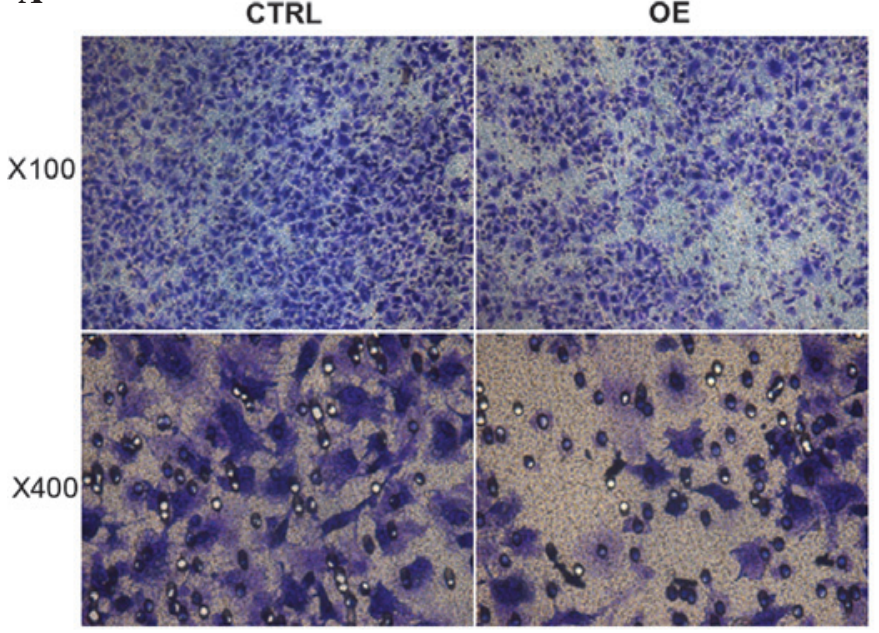

B

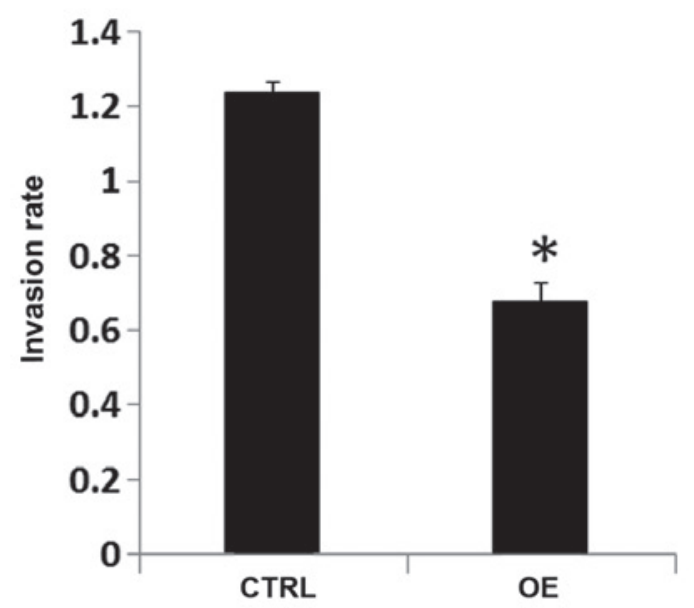

Figure 4. Migration of ASPC-1 pancreatic cancer cells under hypoxic conditions. (A) Migration of ASPC-1 pancreatic cancer cells was observed under a microscope. Cells were stained with Giemsa as described in Materials and methods. (B) The invasion rate was determined by using a microplate reader as described in Materials and methods. Representative results of three independent experiments are shown. CTRL, cells transfected with empty vector (negative control); OE, cells double-transfected with PTEN and KAIl genes; ${ }^{*} \mathrm{P}<0.05$, compared with control.

A
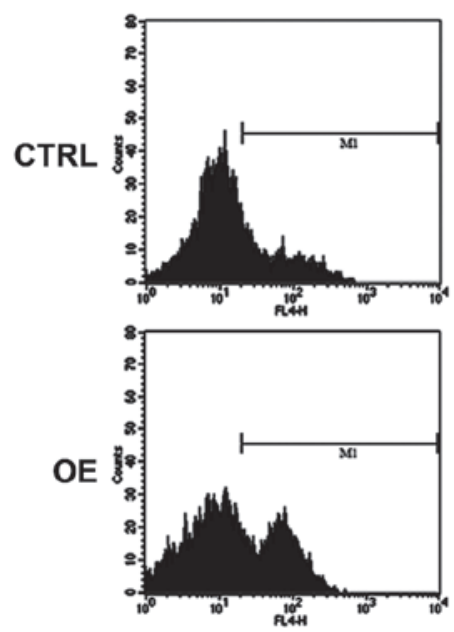

B

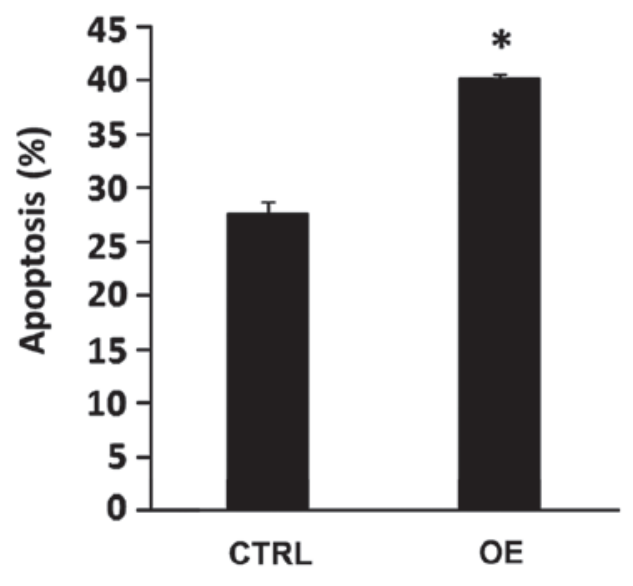

Figure 5. Apoptotic rate of X-ray treated ASPC-1 pancreatic cancer cells under hypoxic conditions. (A) Apoptosis of ASPC-1 cells, which were stably transfected with empty vector or co-transfected with PTEN and KAII genes. (B) Percentage of apoptotic ASPC-1 cells. CTRL, cells transfected with empty vector (negative control); OE, cells double-transfected by PTEN and KAIl genes; ${ }^{\mathrm{P}}<0.05$, compared with control.

that of the control cells $(\mathrm{P}<0.05$; Fig. 5B). This demonstrated that PTEN and KAII genes promoted ASPC-1 cell apoptosis during the X-ray treatment process.

\section{Discussion}

With improvements in lifestyle and an aging population, the morbidity of pancreatic cancer has notably increased worldwide. In 2008, an estimated 37,680 cases of pancreatic cancer were diagnosed in the USA with 34,290 fatalities (11). The clinical manifestation of pancreatic cancer commonly presents as non-specific symptoms, thus the majority of patients are diagnosed with advanced or locally advanced pancreatic cancer, which is unresectable. With the development of radiotherapy, three-dimensional conformal radiotherapy and intensity-modulated radiation therapy have been recently recommended as the main therapeutic approaches for pancreatic cancer. However, the blood supply is commonly uneven during the tumor growth process and thus hypoxia may occur, which reduces the efficacy of radiotherapy, and promotes tumor proliferation and metastasis $(12,13)$. Accounting for these factors, the effect of combination gene therapy on the proliferation and metastasis of pancreatic cancer cells was investigated in the present study.

PTEN is a conservative tumor suppressor gene identified following the identification of p53, which is closely associated with tumor progression. PTEN is located on chromosome 10q23.3, contains nine exons and eight introns, and has been shown to exert a pivotal regulatory role in cell cycle arrest, cell proliferation and possibly cell migration suppression (14-16). Previous studies have demonstrated that the PTEN gene arrested ASPC-1 cell growth at the $\mathrm{G} 2 / \mathrm{M}=$ phase, promoted hypoxia-induced cell apoptosis and $\mathrm{X}$-ray-induced G2/M phase cell arrest, and inhibited ASPC-1 cell proliferation under normoxic and hypoxic conditions $(17,18)$. 
$K A I 1 / \mathrm{CD} 82$, a tumor suppressor gene, was first isolated from a prostate carcinoma cell line in 1995 (4). KAIl is a member of the transmembrane-4 superfamily and encodes a 29.6-kDa transmembrane glycoprotein, which is important in regulating cell motility and differentiation, and inhibiting tumor metastasis (19). Previous studies have demonstrated that the KAIl gene is closely associated with pancreatic cancer metastasis; pancreatic cancer cell growth and migratory ability were significantly restrained following transfection with the KAIl gene (20,21). In vivo studies also revealed significantly reduced lesion metastasis number and size in liver and lung mouse tumors following injection with a KAII-expressing plasmid, compared with that in the control group (20-22).

Tumor proliferation and metastasis involve an interaction network among multiple genes. Previous studies regarding the regulatory effect of PTEN and KAII transfection on the proliferation and metastasis of pancreatic cancer focused only on single-gene efficacies $(9,22)$. Thus, the effect of combination gene therapy in pancreatic cancer progression and development has not been examined. In addition, the hypoxic conditions that affect the prognosis and treatment sensitivity of pancreatic cancer are rarely investigated. In the present study, ASPC-1 cells double-transfected with PTEN and KAII genes under hypoxic conditions were selected to use in the experiments. Hypoxic conditions partially emulate the natural growth environment of tumor cells. This combination gene therapy may provide a theoretical foundation its use in clinical applications.

In conclusion, the results of the present study demonstrated that double transfection with PTEN and KAII genes significantly inhibited ASPC-1 cell proliferation and colony formation, reduced invasion and metastasis, promoted X-ray induced tumor cell apoptosis and improved radiosensitivity. However, further in vivo studies are required to confirm these results.

\section{Acknowledgements}

This study was supported by the Liaoning Province Natural Science Foundation of China (grant no. 201102238) and the Scientific Research Fund of Liaoning Province Education Department (grant no. L2010627).

\section{References}

1. Otrock ZK, Hatoum HA, Awada AH, Ishak RS and Shamseddine AI: Hypoxia-inducible factor in cancer angiogenesis: structure, regulation and clinical perspectives. Crit Rey Oncol Hematol 70: 93-102, 2009.
2. Hill RP, Marie-Egyptienne DT and Hedley DW: Cancer stem cells, hypoxia and metastasis. Semin Radiat Oncol 19: 106-111, 2009.

3. Cosse JP and Michiels C: Tumour hypoxia affects the responsiveness of cancer cells to chemotherapy and promotes cancer progression. Anticancer Agents Med Chem 8: 790-797, 2008.

4. Dong JT, Lamb PW, Rinker-Schaeffer CW, et al: KAI1, a metastasis suppressor gene for prostate cancer on human chromosome 11p11.2. Science 268: 884-886, 1995.

5. Guo X, Friess H, Graber HU, et al: KAI1 expression is up-regulated in early pancreatic cancer and decreased in the presence of metastases. Cancer Res 56: 4876-4880, 1996.

6. Lombardi DP, Geradts J, Foley JF, Chiao C, Lamb PW and Barrett JC: Loss of KAI1 expression in the progression of colorectal cancer. Cancer Res 59: 5724-5731, 1999.

7. Vivanco I and Sawyers CL: The phosphatidylinositol 3-Kinase-AKT pathway in human cancer. Nat Rev Cancer 2: 489-501, 2002.

8. Parsons DW, Wang TL, Samuels Y, et al: Colorectal cancer: mutations in a signalling pathway. Nature 436: 792, 2005.

9. Li H, Yu J, Guo X, et al: Effects of in vitro PTEN transfection on proliferation of human pancreatic cancer ASPC-1 cells. Zhonghua Nei Ke Za Zhi 44: 191-194, 2005 (In Chinese).

10. Wu CY, Yan J, Yang YF, et al: Overexpression of KAI1 induces autophagy and increases MiaPaCa-2 cell survival through the phosphorylation of extracellular signal-regulated kinases. Biochem Biophy Res Commun 404: 802-808, 2011.

11. Ouaïssi M, Julié C, Mitry E, et al: Prognostic factor of recurrence for resected digestive endocrine tumors. Hepatogastroenterology 56: 1183-1189, 2009.

12. McCarthy HO, Worthington J, Barrett E, et al: p21 (WAF1)-mediated transcriptional targeting of inducible nitric oxide synthase gene therapy sensitizes tumours to fractionated radiotherapy. Gene Ther 14: 246-255, 2007.

13. He F, Li L, Kim D, et al: Adenovirus-mediated expression of a dominant negative Ku70 fragment radiosensitizes human tumor cells under aerobic and hypoxic conditions. Cancer Res 67: 634-642, 2007.

14. Chu EC and Tarnawski AS: PTEN regulatory functions in tumor suppression and cell biology. Med Sci Monit 10: RA235-RA241, 2004.

15. Saito Y, Swanson X, Mhashilkar AM, et al: Adenovirus-mediated transfer of the PTEN gene inhibits human colorectal cancer growth in vitro and in vivo. Gene Ther 10: 1961-1969, 2003.

16. Yamada KM and Araki M: Tumor suppressor PTEN: modulator of cell signaling, growth, migration and apoptosis. J Cell Sci 114: 2375-2382, 2001.

17. Li JJ, Li HY, Chen YZ,Li G and Xin Y: Exogenous PTEN enhances apoptosis in pancreas cancer cell line ASPC-1 induced by hypoxia. Zhonghua Zhong Liu Za Zhi 28: 345-348, 2006 (In Chinese).

18. Sridhar SC and Miranti CK: Tetraspanin KAI1/CD82 suppresses invasion by inhibiting integrin-dependent crosstalk with c-Met receptor and Src kinases. Oncogene 25: 2367-2378, 2006.

19. Guo XZ, Xu JH, Liu MP, et al: The mechanism of KAI1 gene in inhibition of metastasis of primary pancreatic cancer. Zhonghua Nei Ke Za Zhi 43: 360-362, 2004 (In Chinese).

20. Xu JH, Guo XZ, Ren LN, Shao LC and Liu MP: KAI1 is a potential target for anti-metastasis in pancreatic cancer cells. World J Gastroenterol 14: 1126-1132, 2008.

21. Guo XZ, Xu JH, Liu MP, et al: KAIl inhibits anchorage-dependent and -independent pancreatic cancer cell growth. Oncol Rep 14: 59-63, 2005.

22. Friess H, Guo XZ, Berberat P, et al: Reduced KAI1 expression in pancreatic cancer is associated with lymph node and distant metastases. Int J Cancer 79: 349-355, 1998. 